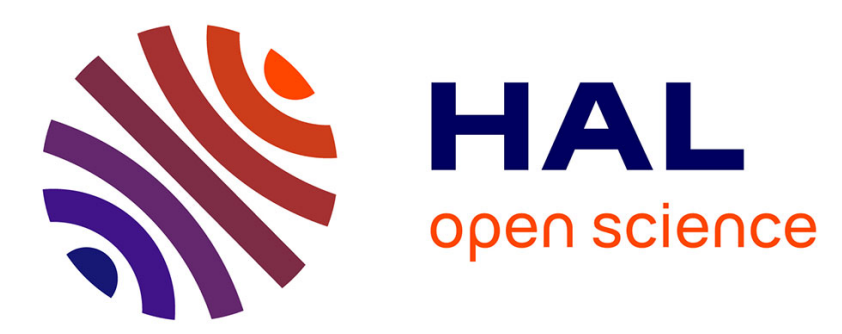

\title{
A Ziv-Zakai type bound for hybrid parameter estimation
}

\author{
Chengfang Ren, Jérôme Galy, Eric Chaumette, Pascal Larzabal, Alexandre \\ Renaux
}

\section{To cite this version:}

Chengfang Ren, Jérôme Galy, Eric Chaumette, Pascal Larzabal, Alexandre Renaux. A Ziv-Zakai type bound for hybrid parameter estimation. ICASSP: International Conference on Acoustics, Speech and Signal Processing, May 2014, Firenze, Italy. 10.1109/ICASSP.2014.6854486 hal-00947790

\section{HAL Id: hal-00947790 \\ https://hal.inria.fr/hal-00947790}

Submitted on 17 Feb 2014

HAL is a multi-disciplinary open access archive for the deposit and dissemination of scientific research documents, whether they are published or not. The documents may come from teaching and research institutions in France or abroad, or from public or private research centers.
L'archive ouverte pluridisciplinaire HAL, est destinée au dépôt et à la diffusion de documents scientifiques de niveau recherche, publiés ou non, émanant des établissements d'enseignement et de recherche français ou étrangers, des laboratoires publics ou privés. 


\title{
A ZIV-ZAKAÏ TYPE BOUND FOR HYBRID PARAMETER ESTIMATION
}

\author{
Chengfang Ren ${ }^{(1)}$, Jerome Galy ${ }^{(2)}$, Eric Chaumette ${ }^{(3,4)}$ Pascal Larzabal $^{(4)}$ and Alexandre Renaux ${ }^{(1)}$
}

(1) Universite Paris-Sud/LSS

3, Rue Joliot-Curie, 91192 Gif-sur-Yvette, France. Emails: cren@1ss.supelec.fr,renaux@1ss.supelec.fr

(2) Universite de Montpellier 2/LIRMM

161 rue Ada 34392 Montpellier Cedex 5, France. Email: galy@lirmm.fr

(3) ONERA/The French Aerospace Lab

Chemin de la Huniere Palaiseau Cedex 91123, France. Email: eric.chaumette@onera.fr

(4) Ecole Normale Superieure de Cachan/SATIE

61 av. du President Wilson, 94235 Cachan cedex, France. Email: pascal.larzabal@ satie.ens-cachan.fr

\begin{abstract}
In statistical signal processing, hybrid parameter estimation refers to the case where the parameters vector to estimate contains both non-random and random parameters. In this communication, we propose a new hybrid lower bound which, for the first time, includes the Ziv-Zakaï bound well known for its tightness in the Bayesian context (random parameters only). For the general case of parameterized mean model with Gaussian noise, closed-form expressions of the proposed bound are provided.
\end{abstract}

Index Terms - Parameter estimation, Ziv-Zakaï bounds, hybrid bounds, SNR threshold

\section{INTRODUCTION}

While Bayesian or non-Bayesian estimation techniques are now widely used in statistical signal processing, the technique called hybrid estimation has been developped more recently and suffers from a relative lack of results. Hybrid parameters refer to the case where the parameters vector to estimate contains both non-random and random parameters with a priori known probability density functions (p.d.f.). Such framework is useful for several signal processing applications, but it is not just the simple concatenation of Bayesian and non-Bayesian techniques. Indeed, new estimator has to be derived and one cannot use the Maximum Likelihood estimator for the non-Bayesian part and the Maximum A Posteriori estimator for the Bayesian part since the parameters can have a dependence. In the same way, performance analysis methods of such hybrid estimators has to be modifed accordingly.

Signal processing community generally use the Hybrid CramérRao Bound (HCRB) [1] for which some asymptotic achievability results [2] are known. The HCRB, as well as the classical CRB, is known to be simple to obtain for various problems (see Part III of [3]) but suffers from some drawbacks. The main one is its only asymptotic tightness in terms of number of samples or Signal-toNoise Ratio (SNR) leading to the incapability of predicting the so-

This work has been partly funded by the European Network of excellence NEWCOM\# called threshold effect (i.e. large errors) on estimator mean square error in non-linear estimation problems. In order to fill this lack, other hybrid lower bounds have already been proposed, e.g. the Hybrid Barankin Bound (HBB) [4] or the Hybrid Barankin/WeissWeinstein bound (HBWWB) [5]. In each case, the key idea is to combine, in a tricky way, some lower bounds already known in the Bayesian and non-Bayesian framework. So far this combination has been done by resorting to the covariance inequality principle which seems to be the cornerstone to establish such hybrid lower bounds $[5][6]$.

However, if we focus our attention on the Bayesian context (random parameter only), two families of bounds are known in the literature: the Weiss-Weinstein family and the Ziv-Zakaï family. If the Weiss-Weinstein family is based on the covariance inequality principle, leading to a natural extension to the hybrid context [5], the Ziv-Zakaï family [7] is based on a binary hypothesis testing problem not a priori linked to the covariance inequality principle. A first consequence is the necessity to resort to simulation in order to compare the tightness of these two families. A second one is the non exhibition of an hybrid lower bound using one of the Ziv-Zakaï family bounds. This paper aims to fill this lack since the Ziv-Zakai bounds are known to be very tight in the Bayesian context (see e.g. [8][9][10]).

First, by adapting an idea suggested in [11, p. 38], we propose an inequality between the hybrid MSE of a general class of estimators and a quantity, closely related to the Ziv-Zakaï bound. We prove that this quantity is independent of the estimation scheme and is, consequently a new lower bound. Moreover, any lower bound on the MSE is a useful bound for signal processing problems if one are able to obtain closed-form expression for a large set of estimation problems. Therefore, in the second part of this paper we derive closed-form expressions of the proposed bound for the general case of Gaussian observation model with parameterized mean. This model is widely met in signal processing problems such that: spectral analysis [12], array processing [13], digital communications [14], etc. Finally, a comparison with the Maximum A Posteriori / Maximum Likelihood Estimator (MAPMLE) and existing bounds is given in a frequency estimation problem. 


\section{RELATION TO PRIOR WORK}

In the Bayesian context, the Weiss-Weinstein bound [15] and the (extended) Ziv-Zakaï bound [7] are both known to be tight while they come from two distinct theories with no a priori relationship. In the hybrid context, a bound including the Weiss-Weinstein bound for the random part has already been proposed [5]. The purpose of the present paper is to provide a hybrid bound including the ZivZakaï bound for the random part.

\section{THE PROPOSED BOUND}

Consider an observation space $\Omega$ of points $\mathbf{X}$ and let $\boldsymbol{\theta}=\left(\theta_{d} \theta_{r}\right)^{T}$ denotes the hybrid parameter vector to estimate where $\theta_{d} \in \Pi_{d} \subseteq \mathbb{R}$ is an unknown deterministic parameter and where $\theta_{r} \in \Pi_{r} \subseteq \mathbb{R}$ is an unknown random parameter characterized by a prior p.d.f. which is assumed to be independent of $\theta_{d}$. In other words $f\left(\theta_{r} ; \theta_{d}\right)=$ $f\left(\theta_{r}\right)$. Let $f(\mathbf{X}, \boldsymbol{\theta})=f\left(\mathbf{X}, \theta_{r} ; \theta_{d}\right)$ denote the joint PDF of $\mathbf{X}$ and $\theta_{r}$ parameterized by $\theta_{d}$. For any estimators $\widehat{\boldsymbol{\theta}}=\left(\widehat{\theta}_{d} \widehat{\theta}_{r}\right)^{T}$ and for any $h_{d}$ and $h_{r}$ such that $\theta_{d}+h_{d} \in \Pi_{d}$ and $\theta_{r}+h_{r} \in \Pi_{r}$ satisfying the following assumptions:

1) $\forall \theta_{r} \in \Pi_{r}, f\left(\mathbf{X}, \theta_{r} ; \theta_{d}\right)=0 \Rightarrow f\left(\mathbf{X}, \theta_{r}+h_{r} ; \theta_{d}+h_{d}\right)=0$.

2) $\forall \theta_{r} \in \Pi_{r}, \mathbb{E}_{\mathbf{X} \mid \theta ; \theta_{d}}\left[\widehat{\theta}_{d}\right]=\theta_{d}, \mathbb{E}_{\mathbf{X} \mid \theta_{r} ; \theta_{d}+h_{d}}\left[\widehat{\theta}_{d}\right]=\theta_{d}+h_{d}$.

3) $\forall \theta_{d} \in \Pi_{d}, \mathbb{E}_{\mathbf{X}, \theta_{r} ; \theta_{d}}\left[\widehat{\theta}_{r}-\theta_{r}\right]=0$ and

$\mathbb{E}_{\mathbf{X}, \theta_{r}+h_{r} ; \theta_{d}}\left[\widehat{\theta}_{r}-\left(\theta_{r}+h_{r}\right)\right]=0$.

Then, estimators MSE is bounded by (The proof is given in Appendix)

$$
\mathbb{E}_{\mathbf{X}, \boldsymbol{\theta}}\left[(\widehat{\boldsymbol{\theta}}-\boldsymbol{\theta})(\widehat{\boldsymbol{\theta}}-\boldsymbol{\theta})^{T}\right] \succeq \mathbf{C V}^{-1} \mathbf{C}^{T},
$$

where " $\mathbf{A} \succeq \mathbf{B}$ " means $\mathbf{A}-\mathbf{B}$ is a positive semidefinite matrix. Each element of matrix $\mathbf{V}$ is given by

$$
\begin{gathered}
\{\mathbf{V}\}_{1,1}=\mu\left(\mathbf{h}_{1}\right)-1, \\
\{\mathbf{V}\}_{2,2}=\beta\left(\mathbf{h}_{2}, \mathbf{h}_{2}\right)+\beta\left(-\mathbf{h}_{2},-\mathbf{h}_{2}\right)-2 \beta\left(\mathbf{h}_{2},-\mathbf{h}_{2}\right),
\end{gathered}
$$
and

$$
\{\mathbf{V}\}_{1,2}=\{\mathbf{V}\}_{2,1}=\alpha\left(\mathbf{h}_{1}, \mathbf{h}_{2}\right)-\alpha\left(\mathbf{h}_{1},-\mathbf{h}_{2}\right) .
$$

The matrix $\mathbf{C}$ is given by

$$
\mathbf{C}=\left(\begin{array}{cc}
h_{1 d} & 0 \\
h_{1 r} & h_{2 r} \alpha\left(\mathbf{0}, \mathbf{h}_{2}\right)
\end{array}\right)
$$

Let us set $\mathbf{h}_{1}=\left(h_{1 d} h_{1 r}\right)^{T}$ and $\mathbf{h}_{2}=\left(0 h_{2 r}\right)^{T}$ which are the so called test-points. Finally $\mu(),. \alpha(.,$.$) and \beta(.,$.$) are defined by$

$$
\begin{gathered}
\mu(\mathbf{h})=\mathbb{E}_{\mathbf{X}, \boldsymbol{\theta}}\left[\frac{f^{2}(\mathbf{X}, \boldsymbol{\theta}+\mathbf{h})}{f^{2}(\mathbf{X}, \boldsymbol{\theta})}\right] \\
\alpha\left(\mathbf{h}_{1}, \mathbf{h}_{2}\right)=\mathbb{E}_{\mathbf{X}, \boldsymbol{\theta}}\left[\frac{f\left(\mathbf{X}, \boldsymbol{\theta}+\mathbf{h}_{1}\right)}{f(\mathbf{X}, \boldsymbol{\theta})} \min \left(\frac{f\left(\mathbf{X}, \boldsymbol{\theta}+\mathbf{h}_{2}\right)}{f(\mathbf{X}, \boldsymbol{\theta})}, 1\right)\right]
\end{gathered}
$$

and

$$
\begin{aligned}
& \beta\left(\mathbf{h}_{1}, \mathbf{h}_{2}\right) \\
= & \mathbb{E}_{\mathbf{X}, \boldsymbol{\theta}}\left[\min \left(\frac{f\left(\mathbf{X}, \boldsymbol{\theta}+\mathbf{h}_{1}\right)}{f(\mathbf{X}, \boldsymbol{\theta})}, 1\right) \min \left(\frac{f\left(\mathbf{X}, \boldsymbol{\theta}+\mathbf{h}_{2}\right)}{f(\mathbf{X}, \boldsymbol{\theta})}, 1\right)\right],
\end{aligned}
$$

where $\min (a, b)$ denotes the smallest value taken from $a$ or $b$. Remarks: (i) If $\boldsymbol{\theta}=\theta_{r}$ only (i.e. assuming that $\theta_{d}$ is known), the proposed bound reduces to one particular form of the Ziv-Zakaï family given by (4.17) in [11] chapter 4 .

(ii) Assumption 1) means that the random parameter support can not be a compact interval; for example the proposed bound does not apply for an uniform prior (as the HBB and the HBWWB).

\section{A PRATICAL FORM FOR COMPLEX GAUSSIAN OBSERVATION MODEL WITH PARAMETERIZED MEAN}

Consider the following complex Gaussian observation model with parameterized mean:

$$
\mathbf{x}=\mathbf{g}\left(\theta_{d}, \theta_{r}\right)+\mathbf{n},
$$

where $\mathbf{x} \in \mathbb{C}^{P}$ is a $P$-dimensional observation vector, the function $\mathbf{g}(.,$.$) is defined from \mathbb{R}^{2}$ to $\mathbb{C}^{P}$ in order to model the physical measurement process and $\mathbf{n}$ is the noise assumed to be random complex circular Gaussian with zero mean and covariance matrix $\sigma_{n}^{2} \mathbf{I}_{P}$. Assume that the prior p.d.f. of $\theta_{r}$ is Gaussian centered with variance $\sigma_{\theta_{r}}^{2}$ and does not depend on $\theta_{d}$ and assume that $\mathbf{n}$ and $\theta_{r}$ are statistically independent.

The main challenge to compute the proposed bound is to obtain $\alpha(.,$.$) and \beta(.,$.$) since the expression of \mu(\mathbf{h})$ is already known in [16]:

$$
\mu(\mathbf{h})=\mathbb{E}_{\theta_{r}}\left(e^{\frac{2}{\sigma_{n}^{2}}\|\mathbf{g}(\boldsymbol{\theta}+\mathbf{h})-\mathbf{g}(\boldsymbol{\theta})\|^{2}}\right) .
$$

We start by the expression of $\beta(.,$.$) since it can be directly ob-$ tained from the literature.

\subsection{Expression of $\beta(.,$.}

For all $\boldsymbol{\delta}_{1}=\left(0 \delta_{1}\right)^{T}$ and $\boldsymbol{\delta}_{2}=\left(0 \delta_{2}\right)^{T}$ such that $\delta_{1} \neq \delta_{2}$, $\beta\left(\boldsymbol{\delta}_{1}, \boldsymbol{\delta}_{2}\right)$ is similar to (2) in [17]

$$
\beta\left(\boldsymbol{\delta}_{1}, \boldsymbol{\delta}_{2}\right)=\mathbb{E}_{\theta_{r}}\left(I_{\beta_{1}}+I_{\beta_{2}}+I_{\beta_{3}}+I_{\beta_{4}}\right)
$$

with

$$
\begin{gathered}
I_{\beta_{1}}=F_{\mathcal{N}\left(\mathbf{o}, \frac{\sigma_{n}^{2}}{2} \boldsymbol{\Gamma}\right)}\left(-\frac{\sigma_{n}^{2} b\left(\delta_{1}\right)}{2},-\frac{\sigma_{n}^{2} b\left(\delta_{2}\right)}{2}\right), \\
I_{\beta_{2}}=e^{-\frac{\delta_{2}^{2}+2 \theta_{r} \delta_{2}}{2 \sigma_{\theta_{r}}^{2}}} F_{\mathcal{N}\left(\mathbf{m}_{\beta_{2}}, \frac{\sigma_{n}^{2}}{2} \boldsymbol{\Gamma}^{\prime}\right)}\left(-\frac{\sigma_{n}^{2} b\left(\delta_{1}\right)}{2}, \frac{\sigma_{n}^{2} b\left(\delta_{2}\right)}{2}\right), \\
I_{\beta_{3}}=e^{-\frac{\delta_{1}^{2}+2 \theta_{r} \delta_{1}}{2 \sigma_{\theta_{r}}^{2}}} F_{\mathcal{N}\left(\mathbf{m}_{\beta_{3}}, \frac{\sigma_{n}^{2}}{2} \boldsymbol{\Gamma}^{\prime}\right)}\left(\frac{\sigma_{n}^{2} b\left(\delta_{1}\right)}{2},-\frac{\sigma_{n}^{2} b\left(\delta_{2}\right)}{2}\right),
\end{gathered}
$$

and

$$
\begin{aligned}
I_{\beta_{4}}= & e^{\frac{2 \operatorname{Re}\left(\mathbf{d}^{H}\left(\delta_{1}\right) \mathbf{d}\left(\delta_{2}\right)\right)}{\sigma_{n}^{2}}-\frac{\delta_{1}^{2}+\delta_{2}^{2}+2 \theta_{r}\left(\delta_{1}+\delta_{2}\right)}{2 \sigma_{\theta_{r}}^{2}}} \\
& \times F_{\mathcal{N}\left(\mathbf{m}_{\beta_{4}}, \frac{\sigma_{n}^{2}}{2} \boldsymbol{\Gamma}\right)}\left(\frac{\sigma_{n}^{2} b\left(\delta_{1}\right)}{2}, \frac{\sigma_{n}^{2} b\left(\delta_{2}\right)}{2}\right) .
\end{aligned}
$$

where $F_{\mathcal{N}(\mathbf{m}, \mathbf{\Sigma})}(\mathbf{a})$ is defined as the value at the point a of a normal cumulative distribution function parameterized by mean $\mathbf{m}$ and covariance matrix $\boldsymbol{\Sigma}, \mathbf{d}\left(\delta_{1}\right)=\mathbf{g}\left(\theta_{d}, \theta_{r}+\delta_{1}\right)-\mathbf{g}\left(\theta_{d}, \theta_{r}\right)$, $b\left(\delta_{1}\right)=\frac{1}{\sigma_{n}^{2}}\left\|\mathbf{d}\left(\delta_{1}\right)\right\|^{2}+\frac{\delta_{1}^{2}+2 \theta_{r} \delta_{1}}{2 \sigma_{\theta_{r}}^{2}}$, 
$\mathbf{m}_{\beta_{2}}=\left(-\operatorname{Re}\left(\mathbf{d}^{H}\left(\delta_{1}\right) \mathbf{d}\left(\delta_{2}\right)\right)\left\|\mathbf{d}\left(\delta_{2}\right)\right\|^{2}\right)^{T}$,

$\mathbf{m}_{\beta_{3}}=\left(\left\|\mathbf{d}\left(\delta_{1}\right)\right\|^{2}-\operatorname{Re}\left(\mathbf{d}^{H}\left(\delta_{1}\right) \mathbf{d}\left(\delta_{2}\right)\right)\right)^{T}$,

$\mathbf{m}_{\beta_{4}}=\left(\begin{array}{c}\left\|\mathbf{d}\left(\delta_{1}\right)\right\|^{2}+\operatorname{Re}\left(\mathbf{d}^{H}\left(\delta_{1}\right) \mathbf{d}\left(\delta_{2}\right)\right) \\ \left\|\mathbf{d}\left(\delta_{2}\right)\right\|^{2}+\operatorname{Re}\left(\mathbf{d}^{H}\left(\delta_{1}\right) \mathbf{d}\left(\delta_{2}\right)\right)\end{array}\right)$,

$\boldsymbol{\Gamma}=\left(\begin{array}{cc}\left\|\mathbf{d}\left(\delta_{1}\right)\right\|^{2} & \operatorname{Re}\left(\mathbf{d}^{H}\left(\delta_{1}\right) \mathbf{d}\left(\delta_{2}\right)\right) \\ \operatorname{Re}\left(\mathbf{d}^{H}\left(\delta_{1}\right) \mathbf{d}\left(\delta_{2}\right)\right) & \left\|\mathbf{d}\left(\delta_{2}\right)\right\|^{2}\end{array}\right)$ and

$\boldsymbol{\Gamma}^{\prime}=\left(\begin{array}{cc}\left\|\mathbf{d}\left(\delta_{1}\right)\right\|^{2} & -\operatorname{Re}\left(\mathbf{d}^{H}\left(\delta_{1}\right) \mathbf{d}\left(\delta_{2}\right)\right) \\ -\operatorname{Re}\left(\mathbf{d}^{H}\left(\delta_{1}\right) \mathbf{d}\left(\delta_{2}\right)\right) & \left\|\mathbf{d}\left(\delta_{2}\right)\right\|^{2}\end{array}\right)$.

Note that if $\delta_{1}=\delta_{2}, \boldsymbol{\Gamma}$ and $\boldsymbol{\Gamma}^{\prime}$ become singular. However, an analytic expression exists in [17] where (11) is modified with the quantities

$$
I_{\beta_{1}}=F_{\mathcal{N}\left(0, \frac{\sigma_{n}^{2}\left\|\mathbf{d}\left(\delta_{1}\right)\right\|^{2}}{2}\right)}\left(-\frac{\sigma_{n}^{2} b\left(\delta_{1}\right)}{2}\right), I_{\beta_{2}}=I_{\beta_{3}}=0
$$

and

$$
\begin{aligned}
I_{\beta_{4}}= & e^{\frac{2\left\|\mathbf{d}\left(\delta_{1}\right)\right\|^{2}}{\sigma_{n}^{2}}-\frac{\delta_{1}^{2}+2 \theta_{r} \delta_{1}}{\sigma_{\theta_{r}}^{2}}} \\
& \times F_{\mathcal{N}\left(2\left\|\mathbf{d}\left(\delta_{1}\right)\right\|^{2}, \frac{\sigma_{n}^{2}\left\|\mathbf{d}\left(\delta_{1}\right)\right\|^{2}}{2}\right)}\left(\frac{\sigma_{n}^{2} b\left(\delta_{1}\right)}{2}\right) .
\end{aligned}
$$

\subsection{Expression of $\alpha(.,$.}

The definition of $\alpha\left(\mathbf{h}_{1}, \mathbf{h}_{2}\right)$ is given by (7). The main idea is to split integration domains in which the min operator can be substituted by $\frac{f\left(\mathbf{x}, \boldsymbol{\theta}+\mathbf{h}_{2}\right)}{f(\mathbf{x}, \boldsymbol{\theta})}$ or 1 . Thus, we can split $\alpha\left(\mathbf{h}_{1}, \mathbf{h}_{2}\right)$ into two parts, for all $\mathbf{h}_{1}=\left(h_{1 d} h_{1 r}\right)^{T}$ and $\mathbf{h}_{2}=\left(0 h_{2 r}\right)^{T}$

$$
\alpha\left(\mathbf{h}_{1}, \mathbf{h}_{2}\right)=\mathbb{E}_{\theta_{r}}\left(I_{\alpha_{1}}+I_{\alpha_{2}}\right)
$$

with

$$
I_{\alpha_{1}}=\int_{\mathcal{V}_{1}} \frac{f\left(\mathbf{x}, \boldsymbol{\theta}+\mathbf{h}_{1}\right)}{f(\mathbf{x}, \boldsymbol{\theta})} f(\mathbf{x} \mid \boldsymbol{\theta}) d \mathbf{x}
$$

and

$$
I_{\alpha_{2}}=\int_{\mathcal{V}_{2}} \frac{f\left(\mathbf{x}, \boldsymbol{\theta}+\mathbf{h}_{1}\right) f\left(\mathbf{x}, \boldsymbol{\theta}+\mathbf{h}_{2}\right)}{f^{2}(\mathbf{x}, \boldsymbol{\theta})} f(\mathbf{x} \mid \boldsymbol{\theta}) d \mathbf{x},
$$

where

$$
\mathcal{V}_{1}=\left\{\mathbf{x} \in \Omega \mid \frac{f\left(\mathbf{x}, \boldsymbol{\theta}+\mathbf{h}_{2}\right)}{f(\mathbf{x}, \boldsymbol{\theta})} \geq 1\right\}
$$

and

$$
\mathcal{V}_{2}=\left\{\mathbf{x} \in \Omega \mid \frac{f\left(\mathbf{x}, \boldsymbol{\theta}+\mathbf{h}_{2}\right)}{f(\mathbf{x}, \boldsymbol{\theta})}<1\right\} .
$$

Some calculus similar to [17] lead to the following closed-form expressions:

$$
I_{\alpha_{1}}=e^{-\frac{h_{1 r}^{2}+2 \theta_{r} h_{1 r}}{2 \sigma_{\theta_{r}}^{2}}} F_{\mathcal{N}\left(m_{\alpha_{1}}, \frac{\sigma_{n}^{2}\left\|\mathbf{d}\left(h_{2 r}\right)\right\|^{2}}{2}\right)}\left(-\frac{\sigma_{n}^{2} b\left(h_{2 r}\right)}{2}\right)
$$

and

$$
\begin{aligned}
I_{\alpha_{2}}= & e^{\frac{2 \operatorname{Re}\left(\tilde{\mathbf{d}}^{H}\left(h_{1 d}, h_{1 r}\right) \mathbf{d}\left(h_{2 r}\right)\right)}{\sigma_{n}^{2}}-\frac{h_{1 r}^{2}+h_{2 r}^{2}+2 \theta_{r}\left(h_{1 r}+h_{2 r}\right)}{2 \sigma_{\theta_{r}}^{2}}} \\
& \times F_{\mathcal{N}\left(m_{\alpha_{2}}, \frac{\sigma_{n}^{2}\left\|\mathbf{d}\left(h_{2 r}\right)\right\|^{2}}{2}\right)}\left(\frac{\sigma_{n}^{2} b\left(h_{2 r}\right)}{2}\right),
\end{aligned}
$$

where the means are $m_{\alpha_{1}}=-\operatorname{Re}\left(\tilde{\mathbf{d}}^{H}\left(h_{1 d}, h_{1 r}\right) \mathbf{d}\left(h_{2 r}\right)\right)$ and $m_{\alpha_{2}}=\operatorname{Re}\left(\tilde{\mathbf{d}}^{H}\left(h_{1 d}, h_{1 r}\right) \mathbf{d}\left(h_{2 r}\right)\right)+\left\|\mathbf{d}\left(h_{2 r}\right)\right\|^{2}$, and where $\tilde{\mathbf{d}}\left(h_{1 d}, h_{1 r}\right)=\mathbf{g}\left(\theta_{d}+h_{1 d}, \theta_{r}+h_{1 r}\right)-\mathbf{g}\left(\theta_{d}, \theta_{r}\right)$.

\section{SIMULATION}

To compare the proposed bound with other, one use the same observation model as in [5] (frequency estimation). Consequently, $\mathbf{g}\left(\theta_{d}, \theta_{r}\right)=\theta_{d} \mathbf{b}\left(\theta_{r}\right)$ where $\theta_{d}$ is the amplitude and $\mathbf{b}\left(\theta_{r}\right)=$ $\left[1 e^{j \theta_{r}} e^{j 2 \theta_{r}} \cdots e^{j(P-1) \theta_{r}}\right]^{T}$ is a normalised cisoïd with angular frequency $\theta_{r}$. The scenario is the following: $P=32$, $\theta_{d}=1$ and $\sigma_{\theta_{r}}^{2}=\frac{1}{2}$. From [18], the HCRB is $2 \times 2$ diagonal matrix with entries $\{\mathbf{H C R B}\}_{1,1}=\frac{\sigma_{n}^{2}}{2 P}$ and $\{\mathbf{H C R B}\}_{2,2}=$ $\left(\frac{2 \theta_{d}^{2}}{\sigma_{n}^{2}}\left(\frac{P(P+1)(2 P+1)}{6}-P^{2}\right)+\frac{1}{\sigma_{\theta_{r}}^{2}}\right)^{-1}$. The HBB, the HBWWB which is given in [5] and the proposed bound are computed with $\mathbf{h}_{1} \in[-1 ; 1] \times\{0\}$ where the sampling interval for the first component is $\delta h_{1 d}=0.01$ and $\mathbf{h}_{2} \in\{0\} \times\left[-\frac{3}{2} ; \frac{3}{2}\right]$ where the sampling interval for the second component is $\delta h_{2 r}=\frac{3}{2^{8}}$. Last, the MAPMLE is obtained by searching the best candidate $s \in[0 ; 2]$ and $\theta_{r} \in\left[-\frac{3}{2} ; \frac{3}{2}\right]$ maximizing the joint p.d.f. $f_{\mathbf{x}, \theta_{r} ; \theta_{d}}\left(\mathbf{x}, \theta_{r} ; \theta_{d}\right)$. The empirical MSE of the MAPMLE is assessed with 1000 MonteCarlo trials. We only plot on the figure (1) the HCRB, the HBB,

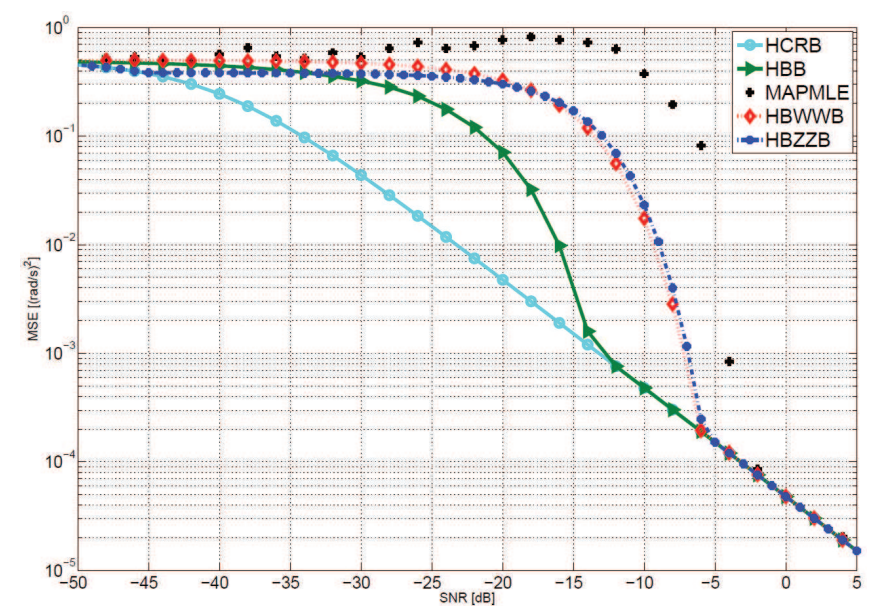

Fig. 1. Comparison of MSE hybrid lower bounds versus SNR

the HBWWB, the proposed bound denoted HBZZB, and the empirical Maximum A Posteriori / Maximum Likelihood Estimator (MAPMLE) MSE for the random parameter $\theta_{r}$ which is the only one exhibiting the threshold effect. We remark that the HBZZB is also accurate to predict the SNR threshold for this estimation problem as well as the HBWWB. Such a comparison has already been seen in the literature but in the Bayesian framework only (see [8] and [19]).

\section{CONCLUSION}

In this paper, a hybrid lower bound on the mean square error based on the Barankin bound and on the Ziv-Zakaï has been developed. As the previously introduced hybrid Barankin Weiss-Weinstein bound, the proposed bound is found to be a tight bound but provide an alternative in terms of calculation. 


\section{APPENDIX}

If $\widehat{\boldsymbol{\theta}}$ is an estimator of $\boldsymbol{\theta}$, from [20, p.124], under some mild regularity assumptions and for any real-valued vector $\mathbf{v}$ with finite second order moment (1) holds. The general expression of matrices involved in (1) are $\mathbf{C}=\mathbb{E}_{\mathbf{X}, \boldsymbol{\theta}}\left[(\widehat{\boldsymbol{\theta}}-\boldsymbol{\theta}) \mathbf{v}^{T}\right]$ and $\mathbf{V}=\mathbb{E}_{\mathbf{X}, \boldsymbol{\theta}}\left[\mathbf{v} \mathbf{v}^{T}\right]$. Note that (1) does not currently lead to a lower bound on the MSE since $\mathbf{C}$ depend on $\widehat{\boldsymbol{\theta}}$. However, let us set $\mathbf{v}=\left(v_{d} v_{r}\right)^{T}$ where $v_{d}=\left\{\begin{array}{l}\frac{f\left(\mathbf{X}, \boldsymbol{\theta}+\mathbf{h}_{1}\right)}{f(\mathbf{X}, \boldsymbol{\theta})}-1 \text { if } \boldsymbol{\theta} \in\{\boldsymbol{\theta}: f(\mathbf{X}, \boldsymbol{\theta})>0, \mathbf{X} \in \Omega\} \\ 0 \text { else }\end{array}\right.$ and $v_{r}=\min \left(\frac{f\left(\mathbf{X}, \boldsymbol{\theta}+\mathbf{h}_{2}\right)}{f(\mathbf{X}, \boldsymbol{\theta})}, 1\right)-\min \left(\frac{f\left(\mathbf{X}, \boldsymbol{\theta}-\mathbf{h}_{2}\right)}{f(\mathbf{X}, \boldsymbol{\theta})}, 1\right)$ for all $\mathbf{h}_{1}=$ $\left(h_{1 d} h_{1 r}\right)^{T}$ and $\mathbf{h}_{2}=\left(0 h_{2 r}\right)^{T}$. By the definition of $\mathbf{V}=$ $\mathbb{E}_{\mathbf{X}, \boldsymbol{\theta}}\left[\mathbf{v v}^{T}\right]$, this choice of $v_{d}$ and $v_{r}$ leads to the matrix $\mathbf{V}$ given in (2), (3) and (4) without major mathematical difficulties. In order to provide a new hybrid lower bound independent of estimation scheme, we have to prove that $\mathbf{C}$ does not depend on $\widehat{\boldsymbol{\theta}}$. It has already be proved that $\{\mathbf{C}\}_{1,1}$ and $\{\mathbf{C}\}_{2,1}$ do not depend on $\widehat{\boldsymbol{\theta}}$ in [5] (see Appendix) under the aforementioned assumptions 2) and 3).

Before calculating $\{\mathbf{C}\}_{1,2}$ and $\{\mathbf{C}\}_{2,2}$, we give a preliminary result: for any real-valued function $l\left(\mathbf{X}, \theta_{d}\right)$ defined on $\Omega \times \Pi_{d}$ and for any $\mathbf{h}=\left(0 h_{r}\right)^{T}$ where $h_{r} \in \Pi_{r}$, one has

$$
\begin{aligned}
& \int_{\Pi_{r}} l\left(\mathbf{X}, \theta_{d}\right)\left(\begin{array}{c}
\min \left(\frac{f(\mathbf{X}, \boldsymbol{\theta}+\mathbf{h})}{f(\mathbf{X}, \boldsymbol{\theta})}, 1\right) \\
-\min \left(\frac{f(\mathbf{X}, \boldsymbol{\theta}-\mathbf{h})}{f(\mathbf{X}, \boldsymbol{\theta})}, 1\right)
\end{array}\right) f(\mathbf{X}, \boldsymbol{\theta}) d \theta_{r} \\
& =l\left(\mathbf{X}, \theta_{d}\right) \int_{\Pi_{r}}\left(\begin{array}{c}
\min (f(\mathbf{X}, \boldsymbol{\theta}+\mathbf{h}), f(\mathbf{x}, \boldsymbol{\theta})) \\
-\min (f(\mathbf{X}, \boldsymbol{\theta}-\mathbf{h}), f(\mathbf{x}, \boldsymbol{\theta}))
\end{array}\right) d \theta_{r} .
\end{aligned}
$$

Note that

$$
\left.\begin{array}{c}
\int_{\Pi_{r}}\left(\begin{array}{c}
\min (f(\mathbf{X}, \boldsymbol{\theta}+\mathbf{h}), f(\mathbf{x}, \boldsymbol{\theta})) \\
-\min (f(\mathbf{X}, \boldsymbol{\theta}-\mathbf{h}), f(\mathbf{x}, \boldsymbol{\theta}))
\end{array}\right) d \theta_{r}= \\
\left(\int_{\Pi^{\prime}}^{\Pi_{r}} \min \left(f\left(\mathbf{X}, \theta_{r}+h_{r} ; \theta_{d}\right), f\left(\mathbf{X}, \theta_{r} ; \theta_{d}\right)\right) d \theta_{r}\right. \\
-\int_{\Pi_{r}}^{\min }\left(f\left(\mathbf{X}, \theta_{r}-h_{r} ; \theta_{d}\right), f\left(\mathbf{X}, \theta_{r} ; \theta_{d}\right)\right) d \theta_{r}
\end{array}\right) .
$$

Let us study the first integral. By substituting $\theta_{r}^{\prime}=\theta_{r}+h_{r}$, the integration domain is still $\Pi_{r}$ by assumption 1 and then,

$$
\begin{aligned}
\int_{\Pi_{r}} & \min \left(f\left(\mathbf{X}, \theta_{r}+h_{r} ; \theta_{d}\right), f\left(\mathbf{X}, \theta_{r} ; \theta_{d}\right)\right) d \theta_{r} \\
\quad= & \int_{\Pi_{r}} \min \left(f\left(\mathbf{X}, \theta_{r}^{\prime} ; \theta_{d}\right), f\left(\mathbf{X}, \theta_{r}^{\prime}-h_{r} ; \theta_{d}\right)\right) d \theta_{r}^{\prime},
\end{aligned}
$$

Thus, using (27) into (25), one obtains

$$
\begin{gathered}
\int_{\Pi_{r}} l\left(\mathbf{X}, \theta_{d}\right)\left(\begin{array}{c}
\min \left(\frac{f(\mathbf{X}, \boldsymbol{\theta}+\mathbf{h})}{f(\mathbf{X}, \boldsymbol{\theta})}, 1\right) \\
-\min \left(\frac{f(\mathbf{X}, \boldsymbol{\theta}-\mathbf{h})}{f(\mathbf{X}, \boldsymbol{\theta})}, 1\right)
\end{array}\right) f(\mathbf{X}, \boldsymbol{\theta}) d \theta_{r} \\
=0 \text { a.e. } \mathbf{X} \in \Omega \text { and for every } \theta_{d} \in \Pi_{d}
\end{gathered}
$$

Remarks:

- This result is similar to condition (1) in [15] with the slight difference that the joint PDF depends on $\theta_{d}$.

- If we chose $\mathbf{h}=\left(h_{d} h_{r}\right)$ with $h_{d} \neq 0$ in this premilinary result, then (28) would depend on $\mathbf{X}$. Consequently, we would find that $\{\mathbf{C}\}_{1,2}$ and $\{\mathbf{C}\}_{2,2}$ would depend on $\widehat{\boldsymbol{\theta}}$. Therefore we use $\mathbf{h}_{2}=\left(0 h_{2 r}\right)^{T}$.
Now, concerning $\{\mathbf{C}\}_{1,2}$, one has

$$
\begin{aligned}
\{\mathbf{C}\}_{1,2} & =\mathbb{E}_{\mathbf{X}, \boldsymbol{\theta}}\left[\left(\widehat{\theta}_{d}-\theta_{d}\right) v_{r}\right] \\
& =\int_{\Omega}\left(\widehat{\theta}_{d}-\theta_{d}\right) \int_{\Pi_{r}}\left(\begin{array}{c}
\min \left(\frac{f\left(\mathbf{X}, \boldsymbol{\theta}+\mathbf{h}_{2}\right)}{f(\mathbf{X}, \boldsymbol{\theta})}, 1\right) \\
-\min \left(\frac{f\left(\mathbf{X}, \boldsymbol{\theta}-\mathbf{h}_{2}\right)}{f(\mathbf{X}, \boldsymbol{\theta})}, 1\right)
\end{array}\right) f(\mathbf{X}, \boldsymbol{\theta}) d \theta_{r} d \mathbf{X} \\
& =0,
\end{aligned}
$$

using (28) with $l\left(\mathbf{X}, \theta_{d}\right)=\widehat{\theta}_{d}-\theta_{d}$

Finally, concerning $\{\mathbf{C}\}_{2,2}$, one has

$$
\begin{aligned}
\{\mathbf{C}\}_{2,2} & =\mathbb{E}_{\mathbf{X}, \boldsymbol{\theta}}\left[\left(\widehat{\theta}_{r}-\theta_{r}\right) v_{r}\right] \\
& =\int_{\Omega} \int_{\Pi_{r}}\left(\widehat{\theta}_{r}-\theta_{r}\right)\left(\begin{array}{c}
\min \left(\frac{f\left(\mathbf{X}, \boldsymbol{\theta}+\mathbf{h}_{2}\right)}{f(\mathbf{X}, \boldsymbol{\theta})}, 1\right) \\
-\min \left(\frac{f\left(\mathbf{X}, \boldsymbol{\theta}-\mathbf{h}_{2}\right)}{f(\mathbf{X}, \boldsymbol{\theta})}, 1\right)
\end{array}\right) f(\mathbf{X}, \boldsymbol{\theta}) d \theta_{r} d \mathbf{X} \\
& =\int_{\Omega} \int_{\Pi_{r}} \theta_{r}\left(\begin{array}{c}
\min \left(f\left(\mathbf{X}, \boldsymbol{\theta}-\mathbf{h}_{2}\right), f(\mathbf{X}, \boldsymbol{\theta})\right) \\
-\min \left(f\left(\mathbf{X}, \boldsymbol{\theta}+\mathbf{h}_{2}\right), f(\mathbf{X}, \boldsymbol{\theta})\right)
\end{array}\right) d \theta_{r} d \mathbf{X},
\end{aligned}
$$

by using (28) with $l\left(\mathbf{X}, \theta_{d}\right)=\widehat{\theta}_{r}$. Let us study

$$
\begin{aligned}
& \int_{\Pi_{r}} \theta_{r} \min \left(f\left(\mathbf{X}, \boldsymbol{\theta}-\mathbf{h}_{2}\right), f(\mathbf{X}, \boldsymbol{\theta})\right) d \theta_{r}= \\
& \int_{\Pi_{r}} \theta_{r} \min \left(f\left(\mathbf{X}, \theta_{r}-h_{2 r} ; \theta_{d}\right), f\left(\mathbf{X}, \theta_{r} ; \theta_{d}\right)\right) d \theta_{r} .
\end{aligned}
$$

By substitution $\theta_{r}^{\prime}=\theta_{r}-h_{2 r}$, the integration domain for $\theta_{r}^{\prime}$ is still $\Pi_{r}$ by assumption 1 and we have

$$
\begin{aligned}
& \int_{\Pi_{r}} \theta_{r} \min \left(f\left(\mathbf{X}, \boldsymbol{\theta}-\mathbf{h}_{2}\right), f(\mathbf{X}, \boldsymbol{\theta})\right) d \theta_{r} \\
& =\int_{\Pi_{r}}\left(\theta_{r}^{\prime}+h_{2 r}\right) \min \left(f\left(\mathbf{X}, \theta_{r}^{\prime} ; \theta_{d}\right), f\left(\mathbf{X}, \theta_{r}^{\prime}+h_{2 r} ; \theta_{d}\right)\right) d \theta_{r}^{\prime} \\
& =\int_{\Pi_{r}} \theta_{r}^{\prime} \min \left(f\left(\mathbf{X}, \theta_{r}^{\prime} ; \theta_{d}\right), f\left(\mathbf{X}, \theta_{r}^{\prime}+h_{2 r} ; \theta_{d}\right)\right) d \theta_{r}^{\prime} \\
& \quad+h_{2 r} \int_{\Pi_{r}} \min \left(f\left(\mathbf{X}, \theta_{r}^{\prime} ; \theta_{d}\right), f\left(\mathbf{X}, \theta_{r}^{\prime}+h_{2 r} ; \theta_{d}\right)\right) d \theta_{r}^{\prime} .
\end{aligned}
$$

Thus, plugging (31) in (29), one has

$$
\{\mathbf{C}\}_{2,2}=h_{2 r} \mathbb{E}_{\mathbf{X}, \boldsymbol{\theta}}\left[\min \left(\frac{f\left(\mathbf{X}, \boldsymbol{\theta}+\mathbf{h}_{2}\right)}{f(\mathbf{X}, \boldsymbol{\theta})}, 1\right)\right] .
$$

Consequently, one has proved that the proposed choice of function $\mathbf{v}$ leads to a matrix $\mathbf{C}$ which does not depend on $\widehat{\boldsymbol{\theta}}$.

\section{REFERENCES}

[1] Y. Rockah and P. Schultheiss, "Array shape calibration using sources in unknown locations-part I: Far-field sources," IEEE Transactions on Acoustics, Speech, and Signal Processing, vol. 35, no. 3, pp. 286-299, Mar. 1987.

[2] Y. Noam and H. Messer, "Notes on the tightness of the hybrid Cramér-Rao lower bound," IEEE Transactions on Signal Processing, vol. 57, no. 6, pp. 2074-2084, 2009.

[3] H. L. Van Trees and K. L. Bell, Eds., Bayesian Bounds for Parameter Estimation and Nonlinear Filtering/Tracking. NewYork, NY, USA: Wiley/IEEE Press, Sep. 2007. 
[4] I. Reuven and H. Messer, "A Barankin-type lower bound on the estimation error of a hybrid parameter vector," IEEE Transactions on Information Theory, vol. 43, no. 3, pp. 1084-1093, May 1997.

[5] C. Ren, J. Galy, E. Chaumette, P. Larzabal, and A. Renaux, "Hybrid lower bound on the mse based on the barankin and weiss-weinstein bounds," in Proc. of IEEE International Conference on Acoustics, Speech, and Signal Processing (ICASSP), Vancouver, Canada, May 2013, pp. 5534-5538.

[6] H. Messer, "The hybrid Cramér-Rao lower bound - from practice to theory," in Proc. of IEEE Workshop on Sensor Array and Multi-channel Processing (SAM), Waltham, MA, USA, Jul. 2006, pp. 304-307.

[7] K. L. Bell, Y. Steinberg, Y. Ephraim, and H. L. Van Trees, "Extended Ziv-Zakaï lower bound for vector parameter estimation," IEEE Transactions on Information Theory, vol. 43, no. 2, pp. 624-637, Mar. 1997.

[8] K. L. Bell, Y. Ephraim, and H. L. Van Trees, "Explicit ZivZakaï lower bound for bearing estimation," IEEE Transactions on Signal Processing, vol. 44, no. 11, pp. 2810-2824, Nov. 1996.

[9] P. Ciblat and M. Ghogho, "Ziv-Zakaï bound for harmonic retrieval in multiplicative and additive Gaussian noise," in Proc. of IEEE Workshop on Statistical Signal Processing (SSP), Bordeaux, FR, Jul. 2005, pp. 561-566.

[10] B. Sadler, N. Liu, and Z. Xu, "Ziv-Zakaï bounds on time delay estimation in unknow convolutive random channels," IEEE Transactions on Signal Processing, vol. 58, no. 5, pp. 27292745, May 2010.

[11] K. L. Bell, "Performance bounds in parameter estimation with application to bearing estimation," Ph.D. dissertation, George Mason University, Fairfax, VA, USA, 1995.

[12] L. Knockaert, "The Barankin bound and threshold behavior in frequency estimation," IEEE Transactions on Signal Processing, vol. 45, no. 9, pp. 2398-2401, Sep. 1997.

[13] B. Ottersten, M. Viberg, P. Stoica, and A. Nehorai, "Exact and large sample maximum likelihood techniques for parameter estimation and detection in array processing," in Radar Array Processing, S. S. Haykin, J. Litva, and T. J. Shepherd, Eds. Berlin: Springer-Verlag, 1993, ch. 4, pp. 99-151.

[14] A. Renaux, "Weiss-Weinstein bound for data aided carrier estimation," IEEE Signal Processing Letters, vol. 14, no. 4, pp. 283-286, Apr. 2007.

[15] E. Weinstein and A. J. Weiss, "A general class of lower bounds in parameter estimation," IEEE Transactions on Information Theory, vol. 34, no. 2, pp. 338-342, Mar. 1988

[16] A. Renaux, P. Forster, P. Larzabal, C. D. Richmond, and A. Nehorai, "A fresh look at the Bayesian bounds of the WeissWeinstein family," IEEE Transactions on Signal Processing, vol. 56, no. 11, pp. 5334-5352, Nov. 2008.

[17] U. Orguner, "Calculation of some expected values for parameterized mean model with Gaussian noise," arXiv:1209.1670v1, 2012.

[18] H. L. Van Trees, Detection, Estimation and Modulation theory: Optimum Array Processing. New-York, NY, USA: John Wiley \& Sons, Mar. 2002, vol. 4.
[19] D. T. Vu, A. Renaux, R. Boyer, and S. Marcos, "Some results on the Weiss-Weinstein bound for conditional and unconditional signal models in array processing," ELSEVIER Signal Processing, vol. 95, pp. 126-148, Feb. 2014.

[20] E. L. Lehmann and G. Casella, Theory of Point Estimation, 2nd ed., ser. Springer Texts in Statistics. New-York, NY, USA: Springer, Sep. 2003. 